\title{
EDITORIAL
}

\section{Polygenic risk scores and their potential clinical use in psychiatry: are we there yet?}

\author{
Gabriel R. Fries ${ }^{1,2,3}$ iD \\ ${ }^{1}$ Translational Psychiatry Program, Faillace Department of Psychiatry and Behavioral Sciences, McGovern Medical School, The University of \\ Texas Health Science Center at Houston, Houston, TX, USA. ${ }^{2}$ Center for Precision Health, School of Biomedical Informatics, The University of \\ Texas Health Science Center at Houston, Houston, TX, USA. ${ }^{3}$ Neuroscience Graduate Program, The University of Texas MD Anderson \\ Cancer Center UTHealth Graduate School of Biomedical Sciences, Houston, TX, USA.
}

The genetics of psychiatric disorders has been known to be extremely complex and heterogeneous for many years. Multiple genome-wide association studies (GWAS) focusing on highly heritable traits (as seen in family and twin studies) have confirmed these disorders to be polygenic and multifactorial -i.e., the result of interactions between environmental stimuli and multiple genetic variants, each of which showing very small effects in determining the risk for the disorder alone. Such variants are believed to include common single nucleotide polymorphisms (SNPs - showing the smallest effects and a typical prevalence of at least $1 \%$ in the population), copy number variants, and rare variants (which have the largest effects), with their specific effects in conferring risk being additive or of higher-order interactions (epistatic). This complex scenario has led to the current understanding that candidate gene studies or single gene markers are not ideal for the field; in fact, the current standard in psychiatric genetics involves the comparison of thousands of genome-wide variants in massive samples of patients and controls collected through consortia (such as the Psychiatric Genomics Consortium [PGC]). Successful examples include the notorious PGC GWAS of schizophrenia, which compared 36,989 cases and 113,075 controls and found 108 loci to be independently associated with the disorder, ${ }^{1}$ and the latest PGC GWAS of bipolar disorder, which compared 20,352 cases and 31,358 controls and identified 30 loci at a genome-wide significance. $^{2}$

As GWAS sample sizes continue to increase, more genome-wide significant variants are expected to be identified in the near future. Nevertheless, strategies to estimate the polygenicity of complex diseases using results from existing GWAS already exist and may provide extremely valuable biomarkers. For instance, a socalled "polygenic risk score" (PRS) can be calculated in subjects from a "target GWAS" by multiplying the number of risk alleles a person has by the effect size of each variant (as detected in an independent "base GWAS"), and then summing each of these products across all risk loci. ${ }^{3}$ Overall, PRS captures the cumulative effects of

Correspondence: Gabriel R. Fries, 1941 East Rd, Suite 3142, 77054, Houston, TX, USA

E-mail: Gabriel.R.Fries@uth.tmc.edu

Submitted Jan 21 2020, accepted Jan 23 2020, Epub Mar 162020. multiple genetic risk variants (or "genetic burden") for a particular phenotype, and often includes thousands of independent SNPs for its calculation (in other words, it includes several SNPs that were only nominally significant in the base GWAS, in addition to the few genomewide significant ones). Typically, PRSs are calculated for many different $p$-value thresholds from the base GWAS, ${ }^{3}$ and the ones that most strongly associate with the phenotype in the target GWAS are chosen for follow-up analyses. This strategy has been used by many research groups, and many recent studies have reported on interesting associations between PRSs calculated for psychiatric disorders and important phenotypes in both patients and controls.

Not surprisingly, the possibilities and implications of such measures are immense. From a theoretical standpoint, PRSs may be used not only to predict a person's risk for a disorder, but also assess its association with specific behavioral, cognitive, and prognostic outcomes. Moreover, PRSs may be used to estimate the genetic correlation between allegedly independent phenotypes, in addition to identifying more homogenous subgroups of patients that may differ in prognostic and treatment aspects based on their genetic burden for specific diseases. Finally, a particularly appealing use of such measures involves the possibility of providing early targeted interventions to disease-free subjects who are found to be at a higher risk of developing a disorder.

Although exciting and while being currently explored in research settings, the clinical applicability of PRS measures is still limited. The main reason for this is the fact that most GWAS for complex diseases, including psychiatric disorders, are still underpowered (of note, the power and accuracy of a particular PRS can only be as high as the power of the original base GWAS that is used for its calculation). Moreover, current GWAS used for PRS calculations rely exclusively on common variants (SNPs), thus not including copy number variations and rare variants that may be extremely important for the heritability of these diseases. In addition, an important limitation that hinders the broader use of PRSs in the 
clinical setting is the lack of ethnic diversity of the GWAS performed for psychiatric disorders to date (the largest PGC studies are overwhelmingly dominated by Caucasian subjects of European descent); however, PRSs are known to be highly sensitive to ethnic background. ${ }^{4}$ Finally, PRSs may not have high specificities due to the known pleiotropic relationships between psychiatric disorders, in addition to the fact that they do not take environmental effects into account. All in all, the consensus is that PRSs are still not very informative at the individual level, and therefore are premature for any clinical use at this moment. ${ }^{4}$

The ability to calculate the genetic burden for psychiatric disorders using PRSs offers many important and clinically significant possibilities. However, it is clear that, at this point, PRSs alone are insufficient to fully capture and explain the genetic risk for these complex and multifactorial conditions. ${ }^{3}$ The discriminatory abilities and clinical applicability of PRSs are expected to significantly improve with more powerful base GWAS, ${ }^{4}$ a focus on studies of diverse ethnic populations, and the development of more sophisticated methods for their calculations. Importantly, given their potential groundbreaking clinical applications, it is imperative that researchers responsibly discuss and explain the limitations of PRSs to the lay public and be aware of the ethical implications these scores may have to society, ${ }^{5}$ including stigmatization and a potentially harmful reductive view of psychiatric disorders.

\section{Acknowledgements}

GRF is supported by a career development grant from the Center for Clinical and Translational Sciences (CCTS), University of Texas Health Science Center at Houston (UTHealth).

\section{Disclosure}

The author reports no conflicts of interest.

\section{References}

1 Schizophrenia Working Group of the Psychiatric Genomics Consortium. Biological insights from 108 schizophrenia-associated genetic loci. Nature. 2014;511:421-7.

2 Stahl EA, Breen G, Forstner AJ, McQuillin A, Ripke S, Trubetskoy V, et al. Genome-wide association study identifies 30 loci associated with bipolar disorder. Nat Genet. 2019;51:793-803.

3 Martin AR, Daly MJ, Robinson EB, Hyman SE, Neale BM. Predicting polygenic risk of psychiatric disorders. Biol Psychiatry. 2019;86:97-109.

4 Fullerton JM, Nurnberger JI. Polygenic risk scores in psychiatry: will they be useful for clinicians? F1000Res. 2019;8.

5 Palk AC, Dalvie S, de Vries J, Martin AR, Stein DJ. Potential use of clinical polygenic risk scores in psychiatry - ethical implications and communicating high polygenic risk. Philos Ethics Humanit Med. 2019;14:4. 